\title{
Use of Nonlocal Spectral for the Spatial Structured Sparse Representation of the Hyper spectral Imagery Restoration
}

\author{
Nikhil R. Kumbhar ${ }^{1}$, Pratima P. Gumaste ${ }^{2}$ \\ Department of E\&TC Engineering, JSP Ms, Jayawantrao Sawant College of Engg, Hadapsar, Pune, India ${ }^{1,2}$
}

\begin{abstract}
In this paper, a sparse representation of noise reduction method for hyper spectral imagery is developed, which is dependent on the assumption that the non-noise component in an observed signal can be sparsely decomposed over a redundant dictionary while the noise component does not have this property. Non locality means the selfsimilarity of image, by which a whole image can be partitioned into some groups containing similar patches. The similar patches in each group are sparsely represented with a shared subset of atoms in a dictionary making true signal and noise more easily separated.Sparse representation with spectral-spatial structure can exploit spectral and spatial joint correlations of hyper spectral imagery by using 3-D blocks instead of 2-D patches for sparse coding, which also makes true signal and noise more distinguished. Moreover, hyper spectral imagery has both signal independent and signal-dependent noises, so a mixed Poisson and Gaussian noise model is used. In order to make sparse representation be insensitive to the various noise distributions in different blocks, a variance-stabilizing transformation (VST) is used to make their variance comparable.
\end{abstract}

Keywords: Variance-fitting transformation (VFT), noise reduction, nonlocal similarity, sparse representation, variancestabilizing transformation.

\section{INTRODUCTION}

The hyper spectral images have been used for varied application fields. It provides information about each spectral and spatial distribution of distinct objects due to its varied and continuous spectral bands. During the imaging method, varied noises will be added into the Imagery, for time being, from imaging identifier and activity error. What is more, spectral, higher spatial, associated radiometric resolutions of hyper spectral imaging have junction rectifier to an increased impact of noises on the item detection and classification results. Several denoising approaches are applied for noise reduction of the Imagery, which include certain diffusion and filters (low pass, arithmetic filters), and wavelet shrinkage [1]-[4].

The sparse illustration is associated in nursing rising and powerful tool for signal and image compression, noise removal, and classification. It gives information about signals supported the scantiness and redundancy of their representations. It usually assumes that signals like images, may be well resembled in terms of a linear combination of a couple of atoms in an exceedingly wordbook. Within the content of image restoration, the references in an exceeding wordbook talks to a collection of image bases.

The low-dimensional nature of such illustration makes it applicable for process and analyzing these signals. As a result, it's incontestable compelling performance in several applications. Traditionally, noise reduction has been with success applied to one-dimensional acoustic signals and Two-dimensional pictures. It faces some challenges once extended to HSI process and analysis.Normally, reduction of noise is done by using sparse representation on one dimensional acoustic signals and 2D images. HSI being

three dimensional images face problem to use the same method. Band by band processing techniques or pixel by pixel processingtechniques are used in many noise reduction techniques.

Hence due to such an approach there might be a correlation loss between bands or pixels. Now to overcome this loss some techniques working in sparse domain came into picture. In these techniques the important change is that the hyper spectral data is considered to be a three dimensional cube. In the approach [5]-[6], shrinkage of 3D wavelet or positive tucker decomposition is applied for denoising of HSI. The HSI data classification and compression can be done by using a joint venture of spectral and spatial information [7]-[8]. Here in spectral - spatial representation denoising, the 2D patch or 1D line segment is replaced by a 3D block because of the strong relation between the spectral and spatial domain data. However in case of noises, they are found to be independent from each other in the sparse representation. Figure 1 shows the example of interpretation of a 2D patch and a HSI data cube.Local information about the signals is the most important factor on which many noise reduction methods are based. This can be observed in the case of mean and median spatial filters, the pixel which is filtered its gray value relies on a neighboringwindow which is centered at this pixel. While in the case of global filters we consider that image has similar property of signal and noise all over the image and hence when changing from spatial to spectral domain and vice versa there is loss of local details of an image. Hence in the denoising process use of sparse representation is done on small patches (i.e. locally) instead of considering whole image at a go. 


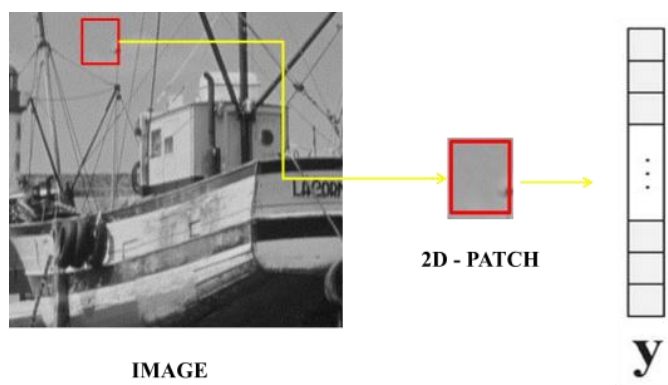

Fig.1. 2D Patch Block.

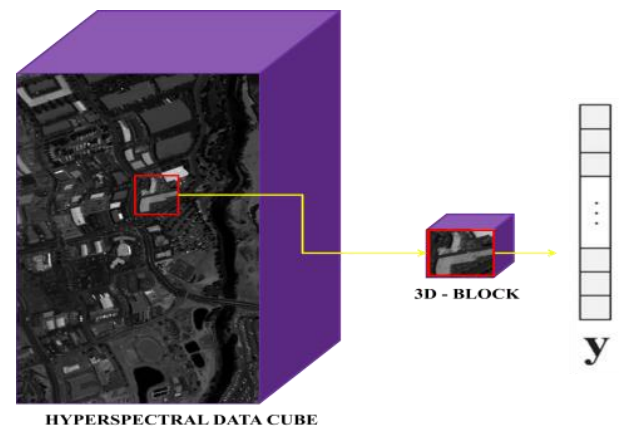

Fig.2. 3D Patch Block.

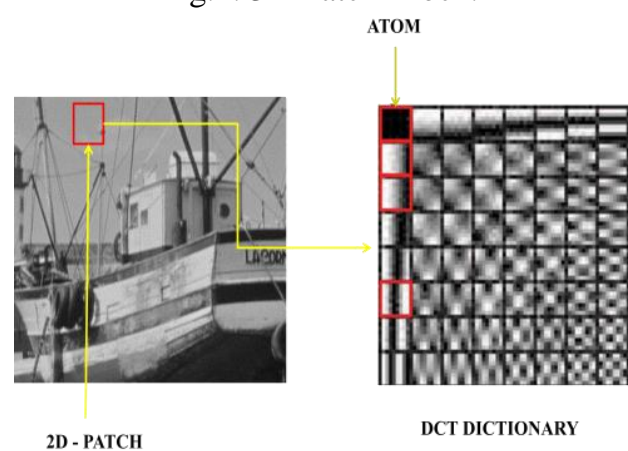

Fig.3. Dictionary Based Sparse Representation

However the neighborhood information limits us to sustain the true structure, details and texture of an image which is a most important drawback of this method.

This fault is nullified using self-similarity or non-locality algorithm which is well known for dynamically investigating statistical and geometric structures of signal and noise. In this we assume that every image has many small patches which have some similarity in them. By using this terminology an algorithm was introduced for noise reduction [9]-[10], in this spatial - spectral representations of non - local patches are re-extracted by using a regular linear regression model with common limitation of sparsity. The assumption used is that a same subset of basis atoms can represent the original signals in these non - local patches however noise cannot satisfy this representation. In HSI also this similarity is present in sparse representation due to which nonlocal spatial spectral structured sparse representation can be generated using dictionaries. Figure3 shows an example of DCT dictionary.

For sparse representation the usable dictionaries are classified into two subclasses: Analytical and Learned. The analytical dictionary depends on the mathematical interpretation of the signal. These include Fourier, Discrete Cosine Transformation (DCT), Discrete Wavelet Transformation (DWT) and Curvelets. When a dictionary is prepared by inferring a set of experimental images is the learned dictionary. Learned dictionary algorithms include PCA, GPCA, K-SVD, ICA and NMF [11].

The noise model and the signal parameters are the most important factors for calculating the performance of a denoising algorithm. In the process of capturing the remote HSI images from space, main noise sources are atmospheric noise and instrumental noise. Here we will consider only the in instrumental noise. So, for sensor there can be further noises can be of two types which are signal- dependent (can also be referred as photonic noise), and signal-independent which will be due to the electronic devices being used [12]-[13]. In order to work with such data we will need a mixed noise model which is PoissonGaussian noise model [14]-[15]. The motive behind this work is to introduce a method of HSI restoration using sparse representation. Overall the work depends on: 1) HSI spectral and spatial joint analysis; 2) Using self similarity concept in HSI; 3) Using wavelet dictionary; and 4) Using mixed noise model (i.e. Poisson - Gaussian noise model) which will calculate the distribution of noise over the HSI bands and the noise parameter.

Expected contribution in this paper would be the transform which can be used to manipulate the statistical properties of the HSI such that it can resemble to that of additive Gaussian noise. By doing this, we will easily be able to handle the mixed Poisson - Gaussian noise. Due to the use of sparse representation based noise reduction in HSI the noise variance is not nearly same for every band. Hence by using the variance-fitting transformation (VFT) we can manipulate the Poisson noise under the mixed noise model [12]-[15].

\section{PROPOSED METHODS}

This section reports the mixed noise model and different parameter estimation used in this paper.

\section{A. Mixed Noise Model}

Generally the signal dependent and signal independent are the two noises that can affect HSI. The noise caused by photons is called photon noise and it resembles to Poisson distribution and hence can be also called Poisson noise. The other source of noise is the signal independent noise which is caused by HSI capturing devices which resembles to Gaussian distribution. Considering both the noises a mixed Poisson - Gaussian noise model is defined for HIS.

$$
y=x+m v_{P}+m v_{G}
$$

where $x$ and $y$ are real and ascertained values respectively. $m v_{G}$ is the Gaussian noise and $m v_{P}$ is the Poisson noise.

Poisson noise measurement of real signal with intensity $x$ can be thought as

$$
z=x+m v_{P}
$$


where $m v_{P}$ depends on the signal intensity $x$ and scalar $a$ which is the level of Poisson noise. For photonic noise, it is normally considered that $(1 / \mathrm{a})(\mathrm{z})$ follow a Poisson distribution $(1 / \mathrm{a})(\mathrm{z}) \sim \mathrm{P}(1 / \mathrm{a}) \mathrm{xwhich}$ shows that its conditional probability density function is $p(z / a-k \mid(1 / a)(x))-(x / a)^{k} e^{-(x / a)} / k$ ! [31]. As per Poisson distribution property, $\{(1 / a)(z)\}=\operatorname{var}\{(1 / a)(z)\}=(1 / a)(x)$ xina definite positionof image can be deterministic, such that

$$
E\left\{\frac{1}{a}(z)\right\}=\frac{1}{a} x+E\left\{\frac{1}{a}\left(m v_{P}\right)\right\}
$$

And

$$
(1 / a)^{2} \operatorname{var}\left\{\left(m v_{P}\right)\right\}=1 / a(x)
$$

Then the Poison noise variance $m v_{P}$ is

$$
\operatorname{var}\left\{\left(m v_{P}\right)\right\}=a x
$$

The zero - mean Gaussian distribution will be satisfied by $m v_{G}$ which is defined as

$$
m v_{G} \sim \mathcal{N}(0, b)
$$

Where Gaussian noise variance is $b$. The total noise variance for two independent noise sources can be obtained by summing their variances.

$$
\operatorname{var}\left(m v_{P}+m v_{G}\right)=\sigma^{2}(x)=a x+b
$$

The HSI sensors Poisson noise distribution parameter is different for band to band while the parameter of Gaussian noise distribution remains same this is because various wavelengths cause various different photon noises however the electronic noise is not affected by the wavelength.

\section{B. Different Parameter Estimation}

Fromequation (7) we can say that Poisson - Gaussian noise model is dependent on two parameters $a$ and $b$, which are obtained from true signal $x$ and $\sigma$ that is its corresponding standard deviation with the help of curve fitting. Now there can be two different categories of signal and noise estimation as spectral or spatial correlation based and non-heterogeneous area based.

The spectral or spatial correlation methods depend on firm local spectral and spatial correlation in HSI. Hence linear representation of one clean area is possible in both spectral and spatial domains. Further to estimate the noises remaining unexplained residuals are to be obtained by performing multiple linear regressions. Non heterogeneous area based methods treat the standard deviations of pixel in the non-heterogeneous areas as the standard deviations of noises. The non-heterogeneous areas can be automatically as well as manually. Now since the parameters differ from band to band in HSI, the nonheterogeneous area based algorithm is used for each band image individually. As the true non heterogeneous areas are difficult to be identified in noisy image, take the band image into wavelet domain by using wavelet transformation to extract smooth regions, and then non heterogeneous areas can be detected by level set segmentation.

Lastly, the parameters calculated $a$ and $b$ are verified by curve fitting method considering random sample consensus and least square to remove extra unnecessary areas.

\section{Variance-Fitting Transformation (VFT)}

Once the noise parameters are estimated from the mixed Poisson - Gaussian model, we will find that there is variance in the noise parameters hence to reduce the noise and its variance VFT is used. It changes Poisson Gaussian noise or Poisson noise into Gaussian noise with an undisturbed noise variance. Its correspondence function is given below. The noise model for pixel by pixel approach can be rewritten as

$$
y=x+v
$$

Where

$$
\operatorname{var}(v)=a x+b
$$

We will have to multiply both the side of equation (9) by $c / \sqrt{a x+b}$ and let $a x+b$ be a constant value $c^{2}$

$c y / \sqrt{a x+b}=c x / \sqrt{a x+b}+c v / \sqrt{a x+b}$

Since $y \approx x$, we get

$$
c y / \sqrt{a y+b}=c x / \sqrt{a x+b}+c v / \sqrt{a x+b}
$$

With this transformation, the observed signal $y$ is associated to ${ }^{c y} / \sqrt{a y+b}$, so the VFT function will be

$$
f_{V F T}(y)=c y / \sqrt{a y+b}
$$

Where gray value of pixel before VFT is $y$ and constant noise deviation after VFT is $c$. Further the inverse VFT can be obtained as

$f_{I V F T}(\tilde{x})=f_{V S T}^{-1}(\tilde{x})=\frac{a \tilde{x}^{2}+\sqrt{a^{2} \tilde{x}^{4}+4 b c^{2} \tilde{x}^{2}}}{2 c^{2}}$

Where denoised gray value of pixel after VFT is $\tilde{x}$, $\mathrm{c}$ is a scale value of noise variance that stabilizes noise variance of $v$ and also changes signal intensity $x$. So if $c$ is large, intensity of pixel in the image after VFT will also be large, and if it is small, the intensity of the pixel after VFT will also be small. Hence we can randomly set the value of $c$ which has no effect on denoising performance in theory. So briefly we can summarize VFT usage as:

- $\quad$ From an HSI estimate the Noise parameters aand b.

Transform HSI data by using VFT on each pixel in the band images using equation (12).

Apply noise reduction method on transformed HSI data. (Refer Section III) 
- Using (13) apply inverse VFT band by band to restore the denoised HSI

\section{Denoising based on sparse representation}

While processing the HSI data for denoising generally a band by band or pixel by pixel approach is followed. This is because it becomes easy for us to use 1D signal processing or $2 \mathrm{D}$ image processing methods for them. However drawback of this is that we can concentrate on either spatial domain or on the spectral domain at one time. Hence nowadays spatial - spectral joint correlation is more attractive. With this sparse representation the concept of non -local similarities also should be considered as an important method for denoising of HIS.

Besides native similarity in image, non native similarity additionally exists, i.e., each little patch in a picture has several similar patches within the same image. We can observe in figure 4 that the patches with constant color boundary square measure terribly just like one another. Non nativedenoising strategies use the correlation between these similar patches to revive truth image. We tend to incorporate non neighborhood into distributed illustration. Non native distributed illustration was originally projected for image restoration then more extended.

This methodology generates the distributed representations of comparable patches at the same time by creating them share constant set of basis atoms. Compared with patchwise distributed denoising methodology within which the chosen set of basis atoms could vary from patch to patch, non native distributed denoising will provides a uniform distributed structure for all similar patches. If we tend to think about generating divided explanation of a patch or block as one task, nonlocal methodology will been seen as a multi-task distributed committal to writing that solves many distributed illustration issues by an optimization performed.

Several researches have shown that the HSI has sturdy correlations in each spatial and spectral domain, therefore we tend to use $3 \mathrm{D}$ blocks rather than second patches within the distributed illustration, it tells us that a HSI cube is categorized into overlapping 3D blocks with the dimensions $n \times n \times n$ as shown in figure 4. Similarly, the dictionary is built from 3D DCT and 3D DWT. The mathematical form of the 3D non local sparse noise reduction model is as follows. We have $N$ similar blocks, then $Y=\left|y_{1}, \ldots, y_{N}\right|$, where $y$ is a column vector of length $n^{2}$ which describes a noise patch and $\mathrm{D}$ is the dictionary matrix given by $D=\left|D_{1}, \ldots, D_{P}\right|$ with $P$ basis atoms. Now the model of multi-task sparse representation is

$\grave{W}=\arg _{W} \min \frac{1}{2}\|Y-D W\|_{F}^{2}+\lambda\|W\|_{1,2}$

Where $\|.\|_{F}$ is the Frobenius norm, and $\|W\|_{1,2}=$ $\sum_{p=1}^{P}\left\|W^{P}\right\|_{2}$ is the $l_{1,2}$ norm, which is the sum of each row's $l_{2}$ - norm. $\lambda$ will be the parameter of regularization which controls the sparsity degree in W. Further the special case of (14) is defined for learning problem as

$$
\min _{W} f(W)+\lambda\|W\|_{1,2}
$$

Where $f($.$) is a differentiable convex loss function. The$ $3 \mathrm{D}$ self-similarity sparse noise reduction method can be summarized as follows:

Categorize HSI cube into overlapping 3D- blocks of size $n \times n \times n$ and every block $B(i, j, k)$ will have centre at voxel $(\mathrm{i}, \mathrm{j}, \mathrm{k})$.

- Considering their similarity further categorize into several groups by K-mean clusteringalgorithm.

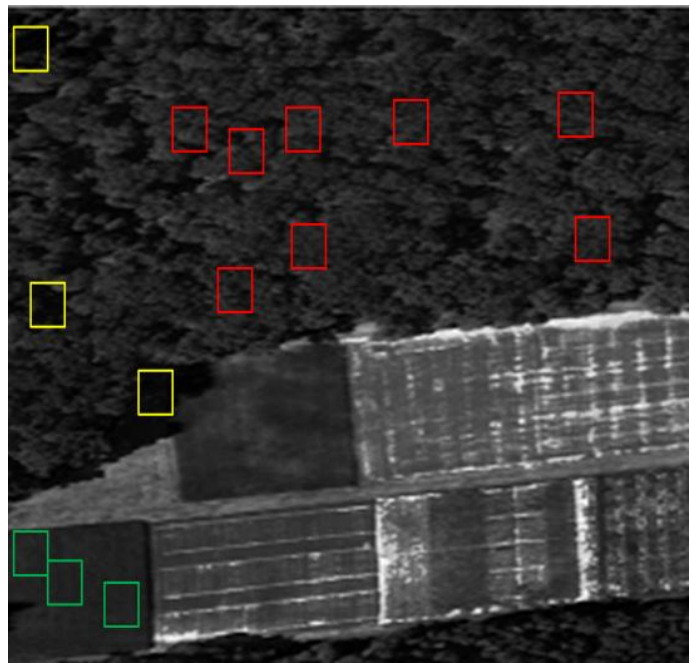

Fig. 4.Self Similarity.

Estimate the real signal from every block in the group by using multi-task sparse coding model.

Using the weighted average method calculate the reduced noise value for each voxel.

\section{III.PROPOSED BLOCK DIAGRAM}

This section describes the proposed block diagram of use of nonlocal spectral for the spatial structured sparse representation of the hyper spectral imagery restoration system. Figure 5 shows the block diagram of proposed system.

For this paper there are total six hyper spectral images are taken for the database. One of these hyper spectral image is given to the noise model in which the Poisson noise and Gaussian noise is to be added in the hyper spectral image with the help of Variance Filtering Transformation (VFT) technique.

The noise added to the hyper spectral image is reduced by using the 3D DWT technique. After that we get noisyhyperspectral image. 


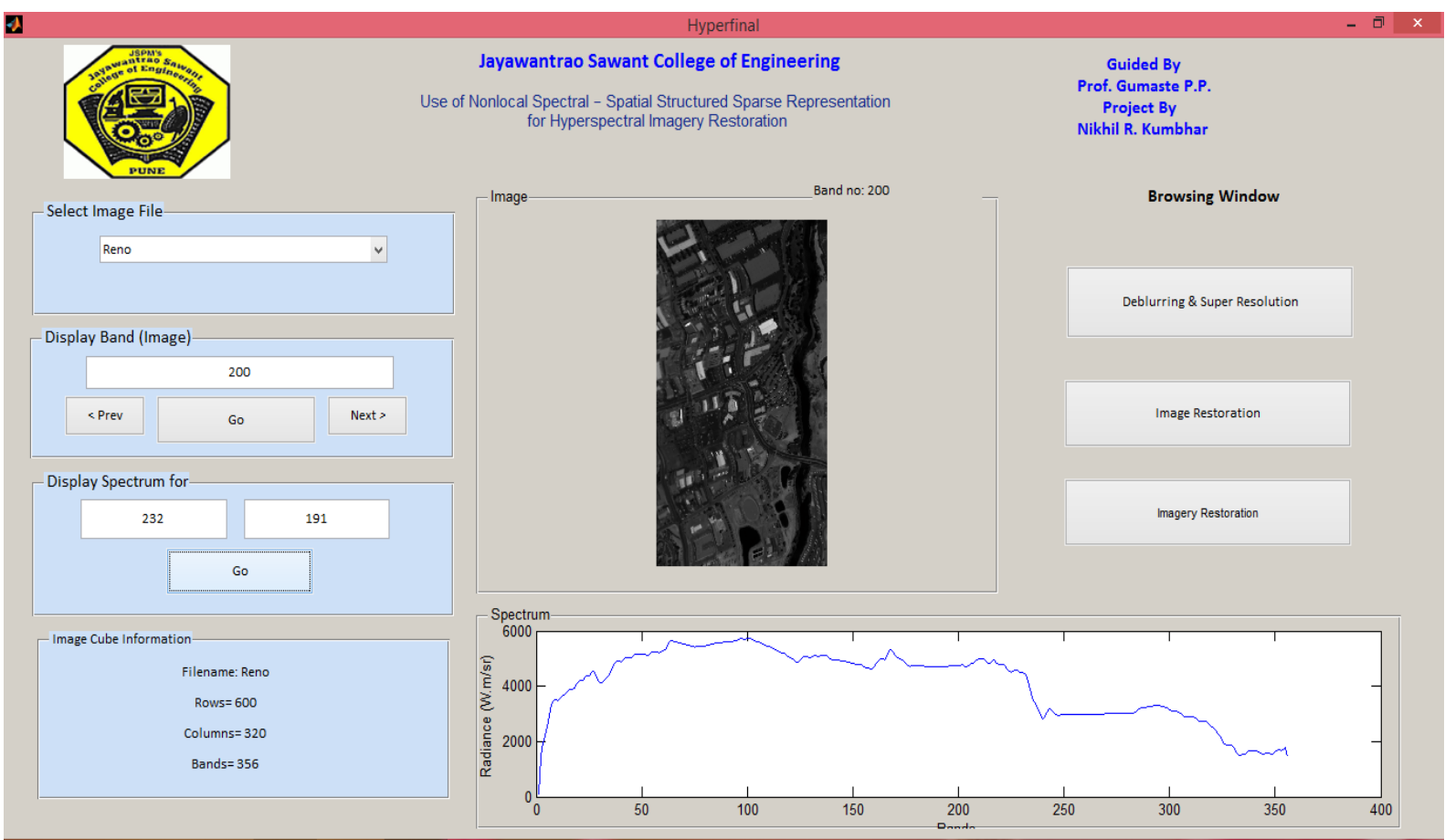

Fig.6.GUI of Proposed System.

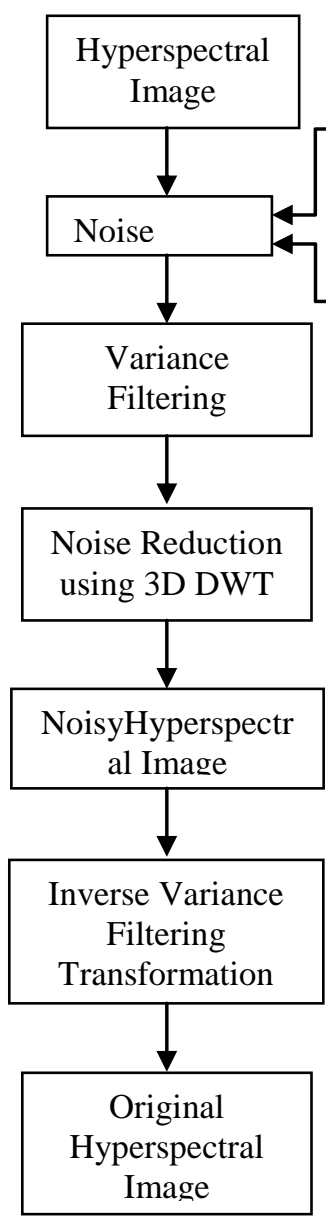

Fig.5. Block Diagram of Proposed Method.

Then by using the Inverse Variance Filtering Fig.7. Reno, Belts, Urban, Terrain, Suwanne and Oil Spill Transformation (IVFT) we remove the noise to get the original hyperspectral image.

\section{IV.EXPERIMENTAL RESULTS}
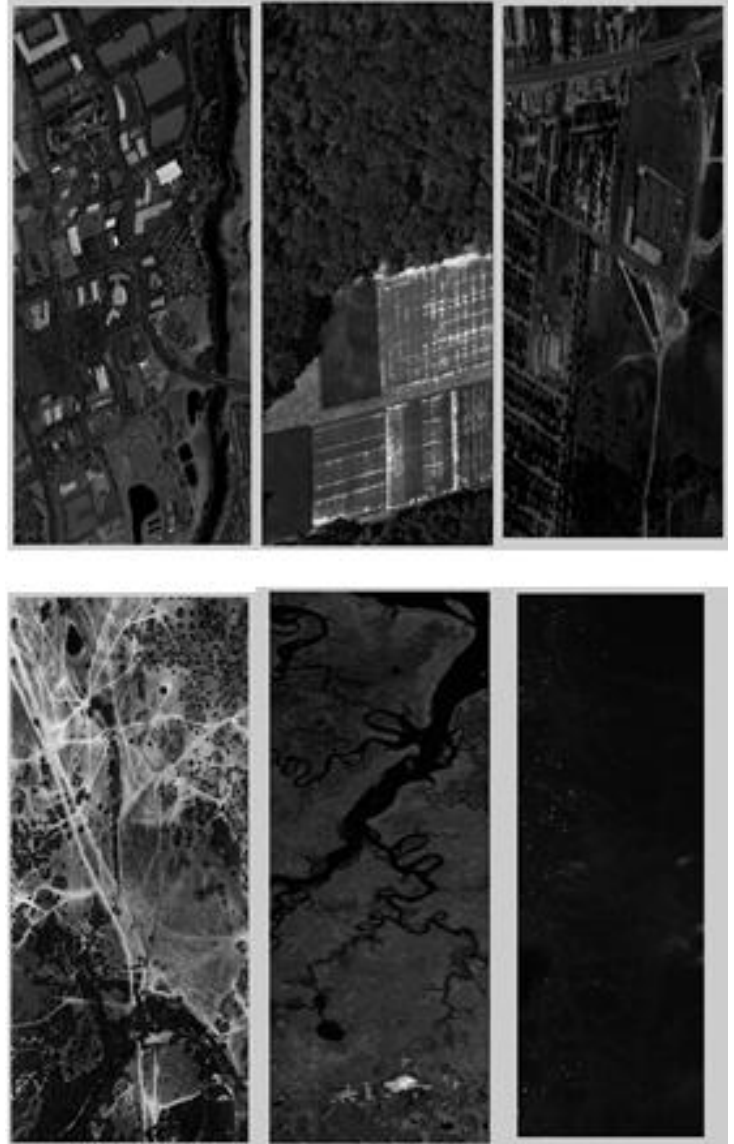


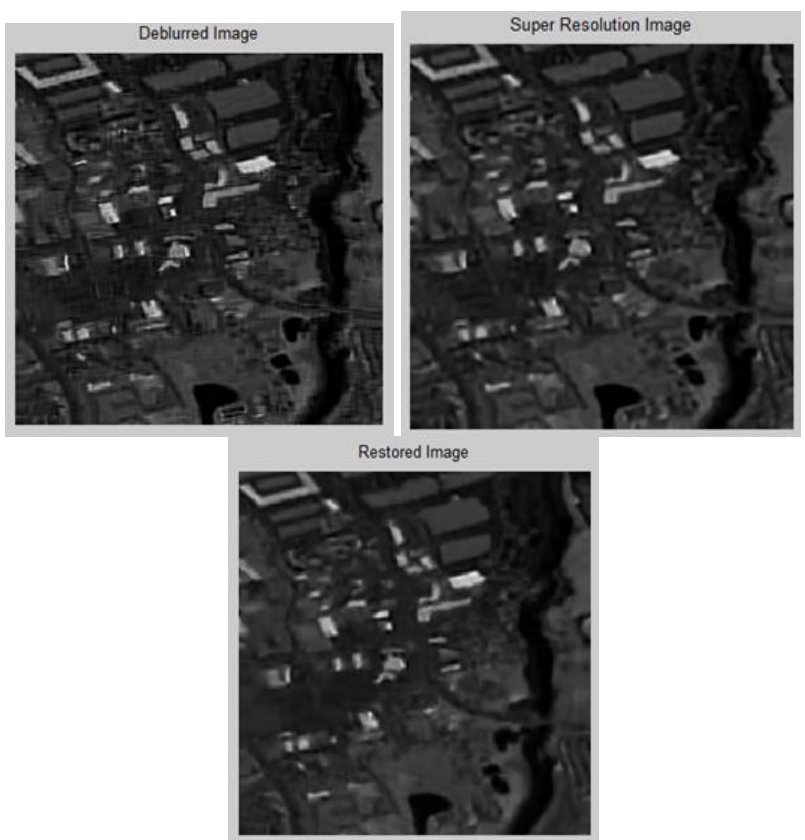

Fig.8.Deblurred Image, Super Resolution image and Restored Image.

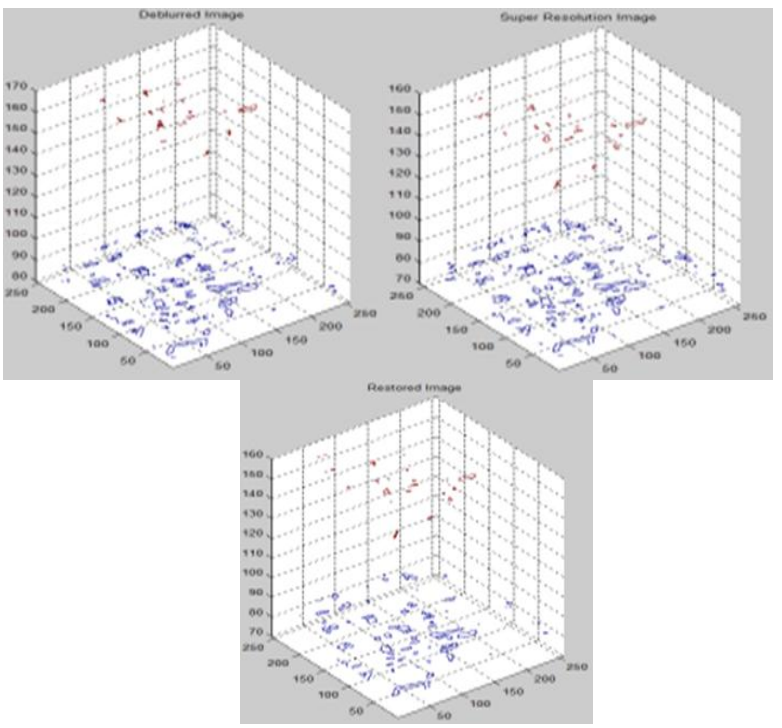

Fig.9.Deblurred Image, Super Resolution image and Restored Image Planes.

This section reports the experimental results of proposed system. In this paper we developed the Graphical User Interface (GUI) in MATLAB. The tool use for developing this proposed work is image processing and wavelet toolbox of MATLAB for "reading" the images and for performing the DWT operations. Figure 6 shows the GUI of Proposed System. Figure 7 shows the different hyper spectral images which are used as data set. Figure 8 shows the deblurred image, super resolution image and restored image. Figure 9 shows the deblurred image, super resolution image and restored image planes.

\section{CONCLUSION}

The previous work done in HSI restoration developed few methods to handle 1D or 2D information of the HSI data. However in these methods correlation between the spatial and spectral bands is not simultaneously considered. Due to which the efficiency of these methods is less. The method discussed in this paper four important points different than that of previous methods of $1 \mathrm{D}$ or $2 \mathrm{D}$ denoising, those are 1) HSI spectral and spatial joint analysis; 2) Using self - similarity concept in HSI; 3) Using wavelet dictionary; and 4) Using mixed noise model (i.e. Poisson - Gaussian noise model) which will calculate the distribution of noise over the HSI bands and the noise parameter. However this method has a drawback that it is based on some prior knowledge of noises and hence can remove only 1 or 2 noises. This may encourage the future work which can be done in order to remove more types of noises from the HSI data. Some research is already being done with the help of Low Rank Matrix (LRM) method for HSI denoising. More accurate denoising of HSI will result in more accurate classification of materials or surfaces which is the main application domain of HSI.

\section{REFERENCES}

[1] R. Phillips, C. Blinn, L. Watson, and R. Wynne, "An adaptive noisefiltering algorithm for AVIRIS data with implications for classification accuracy," IEEE Trans. Geosci. Remote Sens., vol. 47, no. 9., 2009, pp. 3168-3179.

[2] J. Martin-Herrero, "Anisotropic diffusion in the hypercube,'IEEE Trans. Geosci. Remote Sens., vol.45, no.5,2007, pp.1386-1398.

[3] N.Renard, S.Bourennane, and J.Blanc-Talon, "Denoising and dimensionality reduction using multi-linear tools for hyperspectral images," IEEE Geosci. Remote Sens. Lett., vol.5, no.2, 2008, pp.138-14.

[4] G. Chen and S. Qian, "Denoising and dimensionality reduction ofhyperspectral imagery using wavelet packets, neighbour shrinking and principal component analysis," Int. J. Remote Sens., vol. 30, no. 18, 2009, pp. 4889-4895.

[5] J. Wright, Y. Ma, J. Mairal, G. Sapiro, T. Huang, and S. Yan, "Sparse representation for computer vision and pattern recognition," Proc. IEEE, vol. 98, no. 6, 2010, pp. 1031-1044.

[6] M.Jafari and M.Plumbley, "Fast dictionary learning for sparse representations of speech signals," IEEE J. Select. Topics Signal Process., vol. 5, no. 5, 2011, pp. 1025-1031.

[7] A. Karami, M. Yazdi, and A. ZolghadreAsli, "Noise reduction of hyperspectral images using kernel non-negative Tucker decomposition," IEEE J. Select. Topics Signal Process., vol.5, no. 3, 2011, pp.487-493.

[8] B. Rasti, J. R. Sveinsson, M. O. Ulfarsson, and J. A. Benediktsson, "Hyperspectral image denoising using 3D wavelets," in Proc. IEEE Int.Geosci.RemoteSens.Symp.,IGARSS,2012,pp.1349-1352.

[9] E. Christophe, C. Mailhes, and P. Duhamel, "Hyperspectral image compression: adapting SPIHT and EZW to anisotropic 3-D wavelet coding,'IEEETrans.ImageProcess.,vol.17,no.12， 2010， pp.23342346.

[10] Y.Qian, M.Ye, and J.Zhou, "Hyperspectral image classification based on structured sparse logistic regression and three-dimensional wavelet texture features," IEEE Trans. Geosci. Remote Sens., 2012, pp.1-16,

[11] A.Buades, B. Coll, and J.Morel, "A review of imagedenoising algorithms, with a new one," SIAMJ. Multiscale Model. Sim, vol.4, no.2, 2005, pp. 490-530.

[12] R. Wrigley, D. Card, C. Hlavka, J. Hall, F. Mertz, C. Archwamety, and R. Schowengerdt, "Thematicmapper image quality: Registration, noise,andresolution,'IEEE Trans. Geosci. Remote Sens., vol.22, no. 3, 1984, pp. 263-271.

[13] M. Iordache, J. M. Bioucas-Dias, and A. Plaza, "Sparse unmixing of hyperspectraldata,"IEEE Trans. Geosci. RemoteSens., vol.49 ,no.6, 2011, pp. 2014-2039.

[14] M.JafariandM.Plumbley,"Fastdictionarylearningforsparserepresenta tions of speech signals," IEEE J. Select. Topics Signal Process, vol. 5, no. 5, 2011, pp. 1025-1031. 